One- pot oxi dat i ve carbon- car bon bond for mat $i$ on of 3 benzyl ic and 3 al I yl ic indol es with car bon nucl eophi l es

\begin{tabular}{|l|l|}
\hline 著者 & $\begin{array}{l}\text { Nat suo Jun- } \mathrm{i} \text { chi , Tanaki Yumi , I shi bashi } \\
\text { H r oyuki }\end{array}$ \\
\hline $\begin{array}{l}\text { j our nal or } \\
\text { publ i cat i on t i t l e }\end{array}$ & Tet r ahedr on \\
\hline vol une & 64 \\
\hline number & 22 \\
\hline page r ange & $5262-5267$ \\
\hline year & 2005- 05- 01 \\
\hline URL & ht t p: //hdl . handl e. net /2297/9912 \\
\hline
\end{tabular}




\section{Graphical Abstract}

To create your abstract, type over the instructions in the template box below.

Fonts or abstract dimensions should not be changed or altered.

One-pot oxidative carbon-carbon bond formation of
3-benzylic and 3-allylic indoles with carbon
nucleophiles
Jun-ichi Matsuo,* Yumi Tanaki and Hiroyuki Ishibashi*
Division of Pharmaceutical Sciences, Graduate School of Natural Science and Technology, Kanazawa University, Kakuma-machi, Kanazawa 920-1192,
Japan.




\title{
One-pot oxidative carbon-carbon bond formation of 3-benzylic and 3-allylic indoles with carbon nucleophiles
}

\author{
Jun-ichi Matsuo, ${ }^{*}$ Yumi Tanaki and Hiroyuki Ishibashi ${ }^{*}$ \\ Division of Pharmaceutical Sciences, Graduate School of Natural Science and Technology, Kanazawa University, Kakuma-machi, \\ Kanazawa 920-1192, Japan.
}

\begin{abstract}
Indolenines were generated at $-78{ }^{\circ} \mathrm{C}$ from 3-benzylic or 3-allylic indoles by dehydrogenation with $N$-tertbutylbenzenesulfinimidoyl chloride, and a carbon-carbon bond was formed at $-78{ }^{\circ} \mathrm{C}$ in a one-pot manner by treating these indolenines with various carbon nucleophiles such as active methylene compounds or organocuprates. (c) 2008 Elsevier Science. All rights reserved
\end{abstract}

\section{Introduction}

3-Alkylindoles are frequently found as a part of biologically important natural products and pharmaceutical agents, and they have been synthesized by various methods. ${ }^{1,2}$ Since the first report by Snyder in $1944,{ }^{\text {,a }}$ the reaction of carbon nucleophiles with 3-(N,Ndimethylaminomethyl)indole (gramine, 1) or its derivatives has been an important and conventional method for the synthesis of various 3 -substituted indoles (eq. 1). ${ }^{4}$ The C-C bond formation proceeds via reactive indolenines $2^{3 \mathrm{~b}}$ which are usually generated from $\mathbf{1}$ in the presence of nucleophile (e.g. ethyl acetamidomalonate) under several conditions; employment of base at high temperature, ${ }^{3 a, c}$ employment of base and $N$-alkylating agent (e.g. iodomethane and methylsulfate) at room temperature, ${ }^{3 \mathrm{~d}}$ and employment of tri-n-butylphosphine in refluxing acetonitrile. ${ }^{3 \mathrm{e}}$ The similar fragmentation reaction to $\mathbf{2}$ is considered to proceed from isolable intemediates such as 3-(1arylsulfonylalkyl)indole, ${ }^{5} \quad 3$-(alkoxymethyl)indole, ${ }^{3 \mathrm{~b}}$ 3(hydroxymethyl)indole, ${ }^{6}$ 3-(ethylthiomethy)indole, ${ }^{7}$ and 3(1-phenyl- $N$-methylaminomethyl)indole. ${ }^{8}$ Indolenines 2 are believed to be included in a variety of reactions such as synthesis of bisindolylalkanes from indoles and aldehydes. ${ }^{9}$ Also, it is considered that indolenine moiety may be an intermediate in the mechanism of the action of the dehydrogenase enzymes. ${ }^{10}$ Thus, indolenine intermediate works important role in chemical syntheses and in biochemical transformations. It was reported that free indolenines 2 were unstable, but sulfuric acid or hydrogen chloride salts of these indolenines were prepared as stable compounds by reaction of indoles with aromatic aldehydes in the presence of the corresponding acid. ${ }^{11}$

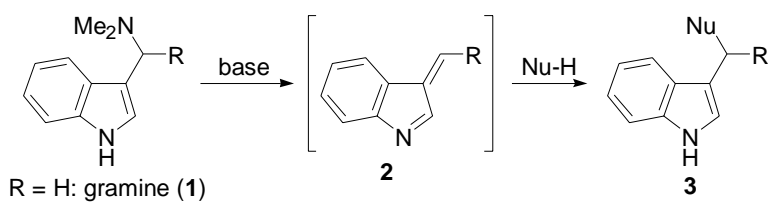

We have been studying mild oxidative C-H activation ${ }^{12}$ of organic molecules with $N$-tert-butylbenzenesulfinimidoyl chloride $(4)^{13}$ to generate a reactive intermediate followed by trapping it with carbon nucleophiles. This method provides a unique $\mathrm{C}-\mathrm{C}$ bond formation at a position where it is difficult to form a new C-C bond by other methods. For example, one-pot oxidative Mannich reactions of $\mathrm{N}$ Cbz amines ${ }^{14 a}$ and lactams ${ }^{14 b}$ and oxidative Michael addition of 1,3-dicarbonyl compounds ${ }^{14 \mathrm{c}}$ have been carried out efficiently. ${ }^{15}$ In the course of our study, we considered the possibility for generating free indolenine intermediate $\mathbf{2}$ by oxidative activation of 3-alkylindole 5 with 4 (Scheme 1). Thus, metallated indole 6 reacts with 4 at the 3-position of indole to afford an intermediate 7 in which elimination should proceed via a five-membered transition state to form free indolenine $\mathbf{2}$ at low temperature. Thus-formed reactive intermediate 2 would react with carbon nucleophile to give indole derivative $\mathbf{3}$ in a one-pot manner. One-pot introduction of a carbon-carbon bond onto the alkyl substituents of 3 -alkylindoles (5 to $\mathbf{3}$ ) is a challenging route

\footnotetext{
* Corresponding author. Tel.: +81-76-234-4439; fax: +81-76-234-4439; e-mail: jimatsuo@p.kanazawa-u.ac.jp.
} 
for derivatizing them, and, to the best of our knowledge, such one-pot bond formation has not been reported to date.

We would like to report here a new method for generating free indolenine $\mathbf{2}$ by dehydrogenation of 3-alkylindoles 5 with 4 at $-78{ }^{\circ} \mathrm{C}$, and unexpectedly high reactivity of indolenine 2 toward carbon nucleophiles such as ethyl cyanoacetate. The reaction profile of organometallic agents toward $\mathbf{2}$ is also described.

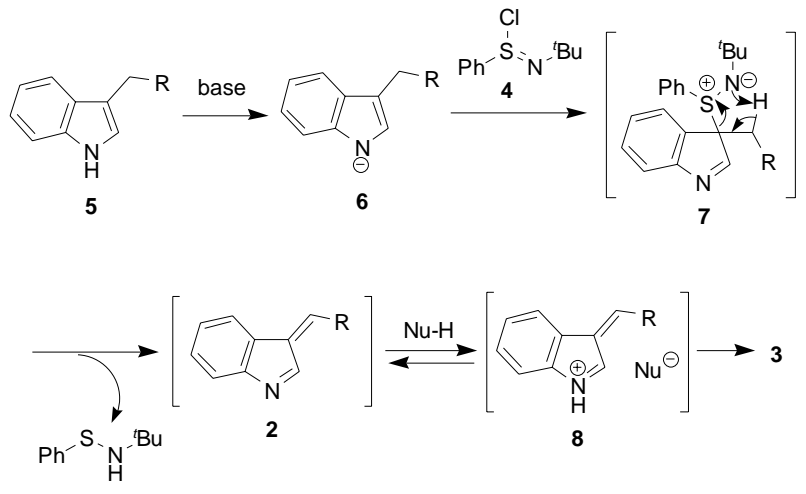

Scheme 1. Generation of indolenine 2 by oxidation of 5 with $\mathbf{4}$ and onepot carbon-carbon bond formation.

\section{Results and discussion}

\subsection{One-pot C-C bond formation of 3-benzylic and 3- allylic indoles with active methylene compounds}

First, suitable reaction conditions for oxidation of 3benzylindole (5a) with 4 were investigated (Table 1). Lithiation of 5a with $n$-BuLi followed by reaction with $\mathbf{4}$ at $-78{ }^{\circ} \mathrm{C}$ proceeded immediately. In order to trap the indolenine intermediate 2a, ethyl cyanoacetate was added. Although gramine derivatives were reported to react with active methylene compounds above at room temperature, ${ }^{3,4}$ the present $\mathrm{C}-\mathrm{C}$ bond formation proceeded at surprisingly low temperature (at $-78{ }^{\circ} \mathrm{C}$ ) to afford adduct 3a in $81 \%$ yield (entry 1 ). Moreover, the intermediate 2a reacted even at $-100{ }^{\circ} \mathrm{C}$ (entry 2). Elevation of reaction temperature from $-78{ }^{\circ} \mathrm{C}$ to room temperature did not improve the yield of 3a. The use of lithium hexamethyldisilazide (LHMDS) instead of $n$-BuLi also gave $\mathbf{3 a}$ in $81 \%$ yield (entry 3). It was noted that lithium ion was superior to sodium or potassium ion as a counter cation of metallated indole $\mathbf{6}$, since sodium hexamethyldisilazide (NHMDS) and potassium hexamethyldisilazide (KHMDS) gave 3a in 61 and 33\% yields, respectively (entries 4 and 5). The effect of metal ion might be explained by regioselectivity (C-3 vs. $\mathrm{N})$ in the reaction of $\mathbf{6}$ and 4 . The use of diisopropylethylamine as a base gave only a trace amount of the desired product 3a (entry 6).
Table 1. Generation of free indolenine $\mathbf{2 a}$ by oxidation of $\mathbf{5 a}$ with $\mathbf{4}$ and successive addition of ethyl cyanoacetate. ${ }^{\text {a }}$

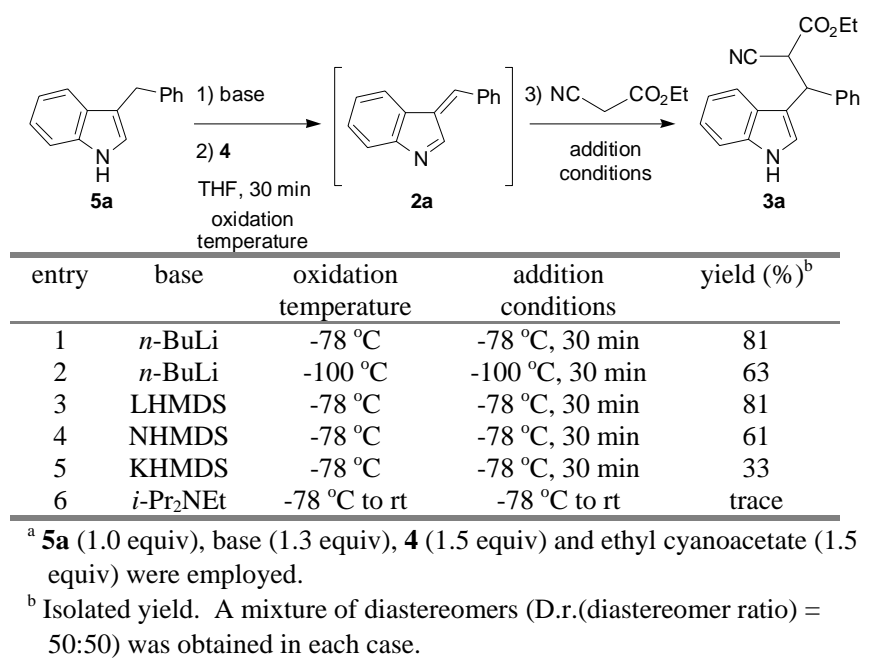

We then investigated the substrate generality of 4-mediated oxidative alkylation of 3-alkylindoles (Table 2). In addition to ethyl cyanoacetate, ethyl allylcyanoacetate and ethyl benzylcyanoacetate reacted to afford the corresponding alkylated products in $83 \%$ and $78 \%$ yields, respectively (entries 1 and 2). Malonic acid esters (entries 3-6), ketoesters (entry 7), and 1,3-diketones (entries 8 and 9) also reacted to give the products in moderate to good yields, whereas the reaction with nitromethane proceeded very sluggishly (12\% yield). Next, several 3-benzylic and 3-allylic indoles were subjected to this oxidative alkylation. 3-(4-Chlorobenzyl)indole (5b) and 3-(4methoxybenzyl)indole (5c) reacted smoothly to afford the corresponding adducts $\mathbf{3 k}$ (78\%) and $\mathbf{3 1}$ (72\%), respectively. 3-Cinnamylindole (5d) also reacted with ethyl cyanoacetate regioselectively to afford $\mathbf{3 m}$ in $84 \%$ yield, while 3allylindole (5e) gave $\mathbf{3 n}$ in $36 \%$ yield along with its regioisomer $3 \mathbf{n}$ ' (11\%). On the other hand, the present oxidative C-C bond formation of 3-methylindole and 3ethylindole with ethy cyanoacetate gave only a trace amount of products. The unprecedented high reactivity of 2 toward active methylene compounds may be ascribed to facilitated attack of carbanion to protonated indolenine $\mathbf{8}$ under neutral conditions (see Scheme 1).

Table 2. Oxidative carbon-carbon bond formation of 3-benzylic and 3allylic indoles with various carbon nucleophiles bearing active methylene or methine protons. 


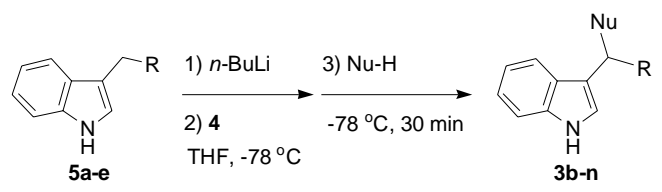

5a: $\mathrm{R}=\mathrm{Ph} ; \mathbf{5 b}: \mathrm{R}=4-\mathrm{ClC}_{6} \mathrm{H}_{4} ; \mathbf{5 c}: \mathrm{R}=4-\mathrm{MeOC}_{6} \mathrm{H}_{4} ; \mathbf{5 d}: \mathrm{R}=\mathrm{CH}=\mathrm{CHPh} ; \mathbf{5 e}: \mathrm{R}=\mathrm{CH}=\mathrm{CH}_{2}$ \begin{tabular}{llll}
\hline entry indol & $\mathrm{NuH}$ & product & yield (\%)
\end{tabular}

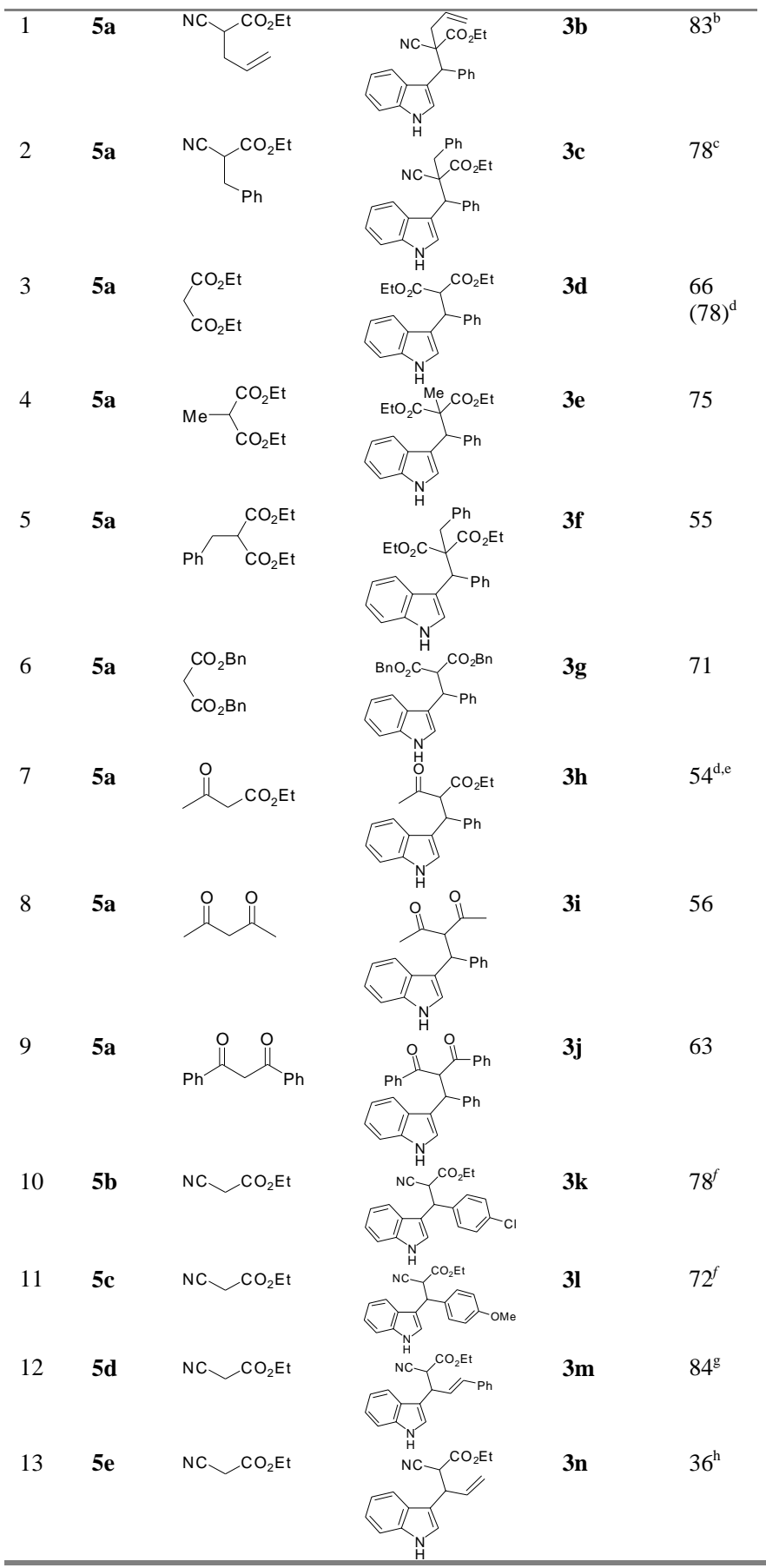

${ }^{\mathrm{a}}$ Isolated yield. ${ }^{\mathrm{b}}$ D.r. $=64: 36$. ${ }^{\mathrm{c}}$ D.r. $=67: 33 .{ }^{\mathrm{d}}$ Determined by ${ }^{1} \mathrm{H}$ NMR analysis using an internal standard. ${ }^{\mathrm{e}}$ D.r. $=56: 44 .{ }^{\mathrm{f}}$ D.r. $=$ 50:50.

${ }^{g}$ D.r. $=60: 40 .{ }^{\mathrm{h}}$ D.r. $=60: 40$. Regioisomer 3n' was also obtained in $11 \%$ yield<smiles>CCOC(=O)C(C#N)C/C=C/c1c[nH]c2ccccc12</smiles>

\subsection{One-pot C-C bond formation of 3-benzylindole (5a) with organometallic agents}

Organometallic agents were next examined as a nucleophile in the present one-pot $\mathrm{C}-\mathrm{C}$ bond formation of 5a. After screening some organometallic reagents, alkyllithium (MeLi, 25\% yield) and Grignard reagents (MeMgBr, 32\% yield; $\mathrm{PhMgBr}$, 35\% yield) were found to be not suitable for the present carbon-carbon bond formation, while alkylzinc ( $\mathrm{Me}_{2} \mathrm{Zn}, 50 \%$ yield; $\mathrm{Et}_{2} \mathrm{Zn}, 70 \%$ yield) gave the alkylated products in moderate yields. It was found that higher-order cyanocuprates gave the desired alkylated and arylated products in good yields (Table 3). Methyl, $n$-butyl, t-butyl, and phenyl groups were introduced smoothly at the benzylic position of $\mathbf{5 a}$.

Table 3. Oxidative carbon-carbon bond formation of $\mathbf{5 a}$ with various higher-order cyanocuprates. ${ }^{a}$

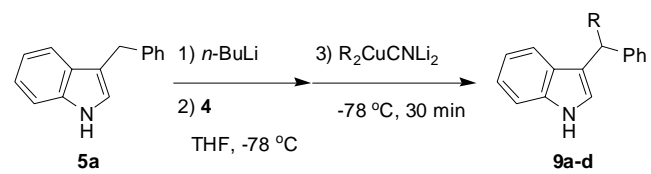

\begin{tabular}{llll}
\hline entry & $\mathrm{R}$ & product & yield $(\%)^{\mathrm{b}}$ \\
\hline 1 & $\mathrm{Me}$ & $\mathbf{9 a}$ & 54 \\
2 & $n-\mathrm{Bu}$ & $9 \mathbf{9 b}$ & 77 \\
3 & $t-\mathrm{Bu}$ & $9 \mathbf{9 c}$ & 54 \\
4 & $\mathrm{Ph}$ & $9 d$ & 82
\end{tabular}

${ }^{a}$ About reaction conditions, see Table 1.

${ }^{\mathrm{b}}$ Determined by ${ }^{1} \mathrm{H}$ NMR analysis.

\section{Conclusion}

3-Benzylic and 3-allylic indoles were activated by oxidation with 4 to form free indolenines 2 at $-78{ }^{\circ} \mathrm{C}$, and various carbon nucleophiles such as active methylene compounds and organocuprates reacted with them at -78 ${ }^{\circ} \mathrm{C}$ in a one-pot manner. The present oxidative activation of indoles with $\mathbf{4}$ will be applicable to various types of nucleophiles and to stereoselective bond formation because of high reactivity observed on $\mathbf{2}$. The present method can be regarded as a new method for generating free indolenine. Recently, $\mathrm{Ir}^{16}$ - or $\mathrm{Pd}^{17}$-catalyzed direct alkylation of indoles with benzylic or allylic alcohols to the corresponding 3alkylindoles has been reported. ${ }^{18}$ Various 3-alkylindoles are now readily prepared, and the present method will be useful for rapid and further derivatization of 3-alkylindoles. Therefore, a diverse chemical library of 3-substituted indoles can be constructed and it would provide an opportunity to discover a more potent drug candidate.

\section{Experimental}

\subsection{General}

All melting points were determined on a Yanagimoto micro melting point apparatus and are uncorrected. Infrared (IR) spectra were recorded on a Shimadzu FTIR-8100. $\mathrm{H}$ 
NMR spectra were recorded on a JEOL JNM EX270 (270 $\mathrm{MHz}$ ) or a JEOL JNM GSX500 (500 MHz) spectrometer; chemical shifts $(\delta)$ are reported in parts per million relative to tetramethylsilane. Splitting patterns are designated as $\mathrm{s}$, singlet; d, doublet; t, triplet; q, quartet; m, multiplet. ${ }^{13} \mathrm{C}$ NMR spectra were recorded on a JEOL JNM GSX500 (500 $\mathrm{MHz}$ ) spectrometer with complete proton decoupling. Chemical shifts are reported in parts per million relative to tetramethylsilane with the solvent resonance as the internal standard $\mathrm{CDCl}_{3}$ and $\mathrm{DMSO}-\mathrm{d}_{6}$. High resolution mass spectra (HRMS) were recorded on a JEOL JMS-SX-102A mass spectrometer. Elemental analyses were carried out on a Yanaco CHN Corder MT-5. Analytical TLC was performed on Merck precoated TLC plates (silica gel 60 GF254, $0.25 \mathrm{~mm}$ ). Silica-gel column chromatography was carried out on silica gel 60N (Kanto Kagaku Co., Ltd., spherical, neutral, 63-210 $\mu \mathrm{m})$. Preparative thin-layer chromatography (PTLC) was carried out on silica gel Wakogel B-5F. THF was distilled under argon from sodium/benzophenone ketyl. All oxidative carbon-carbon bond formations were carried out under argon in dried glassware with magnetic stirring.

$N$-tert-Butylbenzenesufinimidoyl chloride (4) was prepared according to the modified literature procedure ${ }^{19}$ of employing 1.3 equivalents of $N, N$-dichloro-tert-butylamine, and 1 was stored in a refrigerator. 3-Alkylindoles $\left(5 \mathbf{a}^{20}\right.$ $\mathbf{5 b},{ }^{20} \mathbf{5} \mathbf{d},{ }^{17}$ and $5 \mathbf{e}^{17}$ ) were prepared by the reported methods. Ethyl allylcyanoacetate and ethyl benzylcyanoacetate were prepared by alkylation of ethyl cyanoacetate $(t-\mathrm{BuOK}$ and alkylating agent). Other active methylene compounds and methine compounds were purchased and used after distillation.

\subsection{Typical procedure for the oxidative $\mathrm{C}-\mathrm{C}$ bond formation of 3-alkylindole with active methylene compounds (Table 1, entry 1)}

To a stirred solution of 5a (50 mg, $0.241 \mathrm{mmol}$ ) in THF (3 $\mathrm{mL}$ ) was added dropwise a solution of $n$-BuLi (1.65 $N$ in hexane, $0.19 \mathrm{~mL}, 0.31 \mathrm{mmol}$ ) at $-78{ }^{\circ} \mathrm{C}$. After the mixure was stirred for $15 \mathrm{~min}$ at the same temperature, a solution of 4 (77 mg, $0.36 \mathrm{mmol})$ in THF (1 mL) was added at -78 ${ }^{\circ} \mathrm{C}$. After the mixture was stirred for $30 \mathrm{~min}$ at the same temperature, ethyl cyanoacetate $(0.04 \mathrm{~mL}, 0.38 \mathrm{mmol})$ was added at $-78{ }^{\circ} \mathrm{C}$, and the reaction mixture was stirred for 30 min at the same temperature. The reaction mixture was then treated with saturated aqueous $\mathrm{NaHCO}_{3}$, and the resulting mixture was extracted with ethyl acetate (three times). The combined organic extracts were dried over anhydrous $\mathrm{Na}_{2} \mathrm{SO}_{4}$, filtered, and concentrated in vacuo. The crude product was purified by thin layer chromatography on silica gel (hexane/ethyl acetate $=5 / 1$ ) to afford 3a (62 $\mathrm{mg}, 0.195 \mathrm{mmol}, 81 \%$ ) as a white solid.

\subsubsection{2-Cyano-3-(1H-indol-3-yl)-3-phenylpropionic Acid Ethyl Ester (3a) ${ }^{8 b}$}

A mixture of diastereomers (63:27); white solid: mp 79.5$80.5{ }^{\circ} \mathrm{C}$ (hexane-ethyl acetate) [lit. ${ }^{8 b}$ 91-93 ${ }^{\circ} \mathrm{C}$ (from aqueous $\mathrm{AcOH})]$; ${ }^{1} \mathrm{H}$ NMR (500 $\mathrm{MHz}, \mathrm{CDCl}_{3}$, major diastereomer) $\delta 1.10(\mathrm{t}, J=7.5 \mathrm{~Hz}, 3 \mathrm{H}), 4.07-4.20(\mathrm{~m}, 2 \mathrm{H})$, 4.32 (d, $J=6.7 \mathrm{~Hz}, 1 \mathrm{H}), 5.04$ (d, $J=6.7 \mathrm{~Hz}, 1 \mathrm{H}), 7.01-7.08$ (m, 1H), 7.23-7.39 (m, 6H), 7.46 (d, $J=7.3 \mathrm{~Hz}, 2 \mathrm{H}), 8.15$ (s, $1 \mathrm{H}) ;{ }^{1} \mathrm{H}$ NMR (500 MHz, $\mathrm{CDCl}_{3}$, minor diastereomer) $\delta$ 1.08 (t, $J=7.5 \mathrm{~Hz}, 3 \mathrm{H}), 4.07-4.20$ (m, 2H), 4.19 (d, $J=6.7$ $\mathrm{Hz}, 1 \mathrm{H}), 5.10$ (d, $J=6.7 \mathrm{~Hz}, 1 \mathrm{H}), 7.01-7.08$ (m, 1H), 7.157.20 (m, 1H), 7.23-7.39 (m, 6H), 7.50 (d, $J=2.4 \mathrm{~Hz}, 1 \mathrm{H})$, $8.19(\mathrm{~s}, 1 \mathrm{H}) ;{ }^{13} \mathrm{C}$ NMR $\left(67.8 \mathrm{MHz}, \mathrm{CDCl}_{3}\right.$, major diastereomer) $\delta 13.6,43.1,44.2,62.9,111.3,113.1,116.1$, 118.6, 119.6, 122.1, 122.5, 126.5, 127.6, 127.9, 128.6, 136.0, 139.6, 165.2; ${ }^{13} \mathrm{C}$ NMR (67.8 MHz, $\mathrm{CDCl}_{3}$, minor diastereomer) $\delta 13.7,43.1,45.0,62.8,111.3,114.5,116.3$, $118.8,119.7,122.2,122.4,126.0,127.8,128.3,128.6$, 136.2, 138.6, 165.3 .

\subsubsection{2-Allyl-2-Cyano-3-(1H-indol-3-yl)-3- phenylpropionic Acid Ethyl Ester (3b)}

Diastereomers were separated by preparative TLC (hexane/benzene/ethyl acetate $=8 / 8 / 1$ ). The less polar diastereomer; white powder: mp 107.5-108.5 ${ }^{\circ} \mathrm{C}$; ${ }^{1} \mathrm{H}$ NMR $\left(500 \mathrm{MHz}, \mathrm{CDCl}_{3}\right) \delta 0.99(\mathrm{t}, J=7.0 \mathrm{~Hz}, 3 \mathrm{H}), 2.67$ (dd, $J=$ $6.7 \mathrm{~Hz}, 14 \mathrm{~Hz}, 1 \mathrm{H}$ ), 2.79 (dd, $J=7.9 \mathrm{~Hz}, 14 \mathrm{~Hz}, 1 \mathrm{H}$ ), 4.01 (q, $J=7.0 \mathrm{~Hz}, 2 \mathrm{H}), 4.78$ (s, 1H), 5.11-5.15 (m, 2H), 5.72$5.80(\mathrm{~m}, 1 \mathrm{H}), 7.13(\mathrm{t}, J=7.6 \mathrm{~Hz}, 1 \mathrm{H}), 7.20(\mathrm{t}, J=7.3 \mathrm{~Hz}$, 2H), 7.24-7.28 (m, 2H), 7.36 (d, $J=7.9 \mathrm{~Hz}, 1 \mathrm{H}), 7.58$ (d, $J$ $=7.3 \mathrm{~Hz}, 2 \mathrm{H}), 7.64(\mathrm{~d}, J=7.9 \mathrm{~Hz}, 1 \mathrm{H}), 7.71(\mathrm{~d}, J=2.4 \mathrm{~Hz}$, 1H), 8.34 (s, $1 \mathrm{H}) ;{ }^{13} \mathrm{C}$ NMR (125.7 MHz, $\left.\mathrm{CDCl}_{3}\right) \delta 13.7$, 41.7, 48.4, 56.7, 62.5, 111.3, 113.1, 118.1, 119.5, 120.0, 120.1, 122.5, 122.5, 127.5, 127.8, 128.5, 128.6, 130.8, 135.3, 139.5, 168.1; IR $\left(\mathrm{CHCl}_{3}, \mathrm{~cm}^{-1}\right)$ 3021, 2359, 1736, 1211; Anal. Calcd for $\mathrm{C}_{23} \mathrm{H}_{22} \mathrm{~N}_{2} \mathrm{O}_{2}$ : C, 77.07; H, 6.19; N, 7.82. Found: C, 76.74; H, 6.16; N, 7.68.

The more polar diastereomer; white powder: mp 150.5-151 ${ }^{\circ} \mathrm{C}$; ${ }^{1} \mathrm{H}$ NMR (500 MHz, $\left.\mathrm{CDCl}_{3}\right) \delta 0.99$ (t, $\left.J=7.0 \mathrm{~Hz}, 3 \mathrm{H}\right)$, 2.40 (dd, $J=6.7 \mathrm{~Hz}, 13.7 \mathrm{~Hz}, 1 \mathrm{H}$ ), 2.72 (dd, $J=7.9 \mathrm{~Hz}$, $13.7 \mathrm{~Hz}, 1 \mathrm{H}), 4.01$ (q, $J=7.0 \mathrm{~Hz}, 2 \mathrm{H}), 4.78$ (s, $1 \mathrm{H}), 5.16$ (d, $J=4.9 \mathrm{~Hz}, 1 \mathrm{H}), 5.18$ (s, 1H), 5.76-5.82 (m, 1H), 7.00 (t, $J$ $=7.6 \mathrm{~Hz}, 1 \mathrm{H}), 7.12(\mathrm{t}, J=7.0 \mathrm{~Hz}, 1 \mathrm{H}), 7.25-7.36(\mathrm{~m}, 5 \mathrm{H})$, 7.55 (d, $J=6.7 \mathrm{~Hz}, 2 \mathrm{H}), 7.80$ (d, $J=2.4 \mathrm{~Hz}, 1 \mathrm{H}), 8.24$ (s, $1 \mathrm{H}) ;{ }^{13} \mathrm{C}$ NMR $\left(125.7 \mathrm{MHz}, \mathrm{CDCl}_{3}\right) \delta$ 13.7, 42.1, 48.6, 55.3, 62.6, 111.0, 114.2, 118.6, 119.0, 119.6, 120.7, 121.9, 122.4, 126.8, 127.9, 128.6, 129.6, 130.6, 135.5, 138.1, 168.2; IR $\left(\mathrm{CHCl}_{3}, \mathrm{~cm}^{-1}\right)$ 3478, 2359, 1740, 1227; Anal. Calcd for $\mathrm{C}_{23} \mathrm{H}_{22} \mathrm{~N}_{2} \mathrm{O}_{2}$ : C, 77.07; H, 6.19; N, 7.82. Found: C, 76.83; H, 6.18; N, 7.76.

\subsubsection{2-Benzyl-2-Cyano-3-(1H-indol-3-yl)-3- phenylpropionic Acid Ethyl Ester (3c)}

Diastereomers were separated by preparative TLC (benzene/ethyl acetate $=100 / 1)$. The less polar diastereomer; white amorphous: ${ }^{1} \mathrm{H}$ NMR (500 MHz, $\left.\mathrm{CDCl}_{3}\right) \delta 0.81(\mathrm{t}, J=7.2 \mathrm{~Hz}, 3 \mathrm{H}), 3.19(\mathrm{~d}, J=13.6 \mathrm{~Hz}, 1 \mathrm{H})$, 3.36 (d, $J=13.6 \mathrm{~Hz}, 1 \mathrm{H}), 3.79-3.89$ (m, 2H), 4.94 (s, 1H), 7.14-7.26 (m, 10H), $7.38(\mathrm{~d}, J=8.1 \mathrm{~Hz}, 1 \mathrm{H}), 7.61(\mathrm{~d}, J=$ $8.1 \mathrm{~Hz}, 2 \mathrm{H}), 7.72$ (d, $J=8.1 \mathrm{~Hz}, 1 \mathrm{H}), 7.82(\mathrm{~d}, J=2.4 \mathrm{~Hz}$, $1 \mathrm{H}), 8.38$ (s, $1 \mathrm{H})$; ${ }^{13} \mathrm{C}$ NMR (125.7 $\left.\mathrm{MHz}, \mathrm{CDCl}_{3}\right) \delta 13.5$, 43.2, 49.0, 58.6, 62.4, 111.4, 113.2, 118.2, 119.5, 120.0, $122.6,122.7,127.5,127.6,127.9,128.4,128.5,128.7$, 
129.9, 134.5, 135.4, 139.5, 168.0; IR $\left(\mathrm{CHCl}_{3}, \mathrm{~cm}^{-1}\right)$ 3476, 1736; HRMS (EI) Calculated for $\mathrm{C}_{27} \mathrm{H}_{24} \mathrm{~N}_{2} \mathrm{O}_{2}$ : 408.18378 . Found: 408.18390.

The more polar diastereomer; white amorphous: ${ }^{1} \mathrm{H}$ NMR $\left(500 \mathrm{MHz} \mathrm{CDCl}_{3}\right) \delta 0.80(\mathrm{t}, J=7.1 \mathrm{~Hz}, 3 \mathrm{H}), 2.88(\mathrm{~d}, J=$ $13.4 \mathrm{~Hz}, 1 \mathrm{H}), 3.28$ (d, $J=13.4 \mathrm{~Hz}, 1 \mathrm{H}), 3.80-3.89$ (m, $2 \mathrm{H})$, $4.94(\mathrm{~s}, 1 \mathrm{H}), 7.02(\mathrm{t}, J=7.4 \mathrm{~Hz}, 1 \mathrm{H}), 7.13(\mathrm{t}, J=7.6 \mathrm{~Hz}$, $1 \mathrm{H}), 7.20-7.40$ (m, 10H), 7.65 (d, $J=7.3 \mathrm{~Hz}, 2 \mathrm{H}), 7.77$ (d, $J=2.4 \mathrm{~Hz}, 1 \mathrm{H}), 8.14(\mathrm{~s}, 1 \mathrm{H}) ;{ }^{13} \mathrm{C} \mathrm{NMR}(125.7 \mathrm{MHz}$, $\left.\mathrm{CDCl}_{3}\right) \delta 13.5,43.7,49.3,56.9,62.5,111.0,114.3,118.7$, 119.2, 119.7, 121.9, 122.4, 126.9, 127.7, 127.9, 128.4, 128.7, 129.8, 129.8, 134.2, 135.5, 138.2, 168.1; IR $\left(\mathrm{CHCl}_{3}\right.$, $\mathrm{cm}^{-1}$ ) 3478, 2245, 1736, 1242; HRMS (EI) Calculated for $\mathrm{C}_{27} \mathrm{H}_{24} \mathrm{~N}_{2} \mathrm{O}_{2}$ : 408.18378. Found: 408.18410.

\subsubsection{Ethyl 2-[1H-indol-3-yl(phenyl)methyl]malonate $(3 d)^{8 d}$}

White silky filber: mp 183.5-184.0 ${ }^{\circ} \mathrm{C}$ (hexane-ethyl acetate) [ref. ${ }^{8 \mathrm{~d}} 165-167{ }^{\circ} \mathrm{C}$ (from EtOH)]; ${ }^{1} \mathrm{H}$ NMR (500 $\left.\mathrm{MHz} \mathrm{CDCl}_{3}\right) \delta 0.97(\mathrm{t}, J=7.1 \mathrm{~Hz}, 3 \mathrm{H}), 1.00(\mathrm{t}, J=7.1 \mathrm{~Hz}$, $3 \mathrm{H}), 3.95-4.30(\mathrm{~m}, 4 \mathrm{H}), 4.29(\mathrm{~d}, J=11.8 \mathrm{~Hz}, 1 \mathrm{H}), 5.08$ (d, $J=11.8 \mathrm{~Hz}, 1 \mathrm{H}), 7.02(\mathrm{t}, J=7.7 \mathrm{~Hz}, 1 \mathrm{H}), 7.10-7.14(\mathrm{~m}$, 3H), 7.20-7.26 (m, 3H), 7.36 (d, $J=7.6 \mathrm{~Hz}, 2 \mathrm{H}), 7.54$ (d, $J$ $=8.1 \mathrm{~Hz}, 1 \mathrm{H}), 8.09(\mathrm{~s}, 1 \mathrm{H}) ;{ }^{13} \mathrm{C} \mathrm{NMR}\left(125.7 \mathrm{MHz}, \mathrm{CDCl}_{3}\right)$ $\delta 13.7,13.7,42.6,58.4,61.4,61.4,111.0,116.9,119.3$, $119.4,120.9,122.2,126.7,126.7,128.2,128.3,136.2$, 141.4, 167.8, 168.0.

\subsubsection{Ethyl 2-[1H-indol-3-yl(phenyl)methyl]-2- methylmalonate (3e)}

Pale yellow oil: ${ }^{1} \mathrm{H}$ NMR (500 MHz, $\left.\mathrm{CDCl}_{3}\right) \delta 0.93(\mathrm{t}, J=$ $7.0 \mathrm{~Hz}, 3 \mathrm{H}), 1.10$ (t, $J=7.0 \mathrm{~Hz}, 3 \mathrm{H}), 1.66(\mathrm{~s}, 3 \mathrm{H}), 3.91-$ $3.99(\mathrm{~m}, 2 \mathrm{H}), 4.08(\mathrm{q}, J=7.0 \mathrm{~Hz}, 2 \mathrm{H}), 5.36(\mathrm{~s}, 1 \mathrm{H}), 6.99(\mathrm{t}$, $J=7.6 \mathrm{~Hz}, 1 \mathrm{H}), 7.09-7.15$ (m, 2H), 7.18-7.21 (m, 2H), 7.27 (d, $J=7.9 \mathrm{~Hz}, 1 \mathrm{H}), 7.40$ (d, $J=2.4 \mathrm{~Hz}, 1 \mathrm{H}), 7.44$ (dd, $J=$ $7.0 \mathrm{~Hz}, 1.5 \mathrm{~Hz}, 3 \mathrm{H}), 8.11(\mathrm{~s}, 1 \mathrm{H}) ;{ }^{13} \mathrm{C} \mathrm{NMR}(67.8 \mathrm{MHz}$, $\left.\mathrm{CDCl}_{3}\right) \delta 13.5,13.7,19.1,46.7,59.2,61.3,61.3,110.8$, $115.2,119.1,119.2,121.9,122.4,126.5,127.7,127.9$, 130.3, 135.3, 140.2, 171.2, 171.5; IR $\left(\mathrm{CHCl}_{3}, \mathrm{~cm}^{-1}\right)$ 3480, 2350, 1728; HRMS (EI) Calculated for $\mathrm{C}_{23} \mathrm{H}_{25} \mathrm{NO}_{4}$ : 379.17836. Found 379.17920.

\subsubsection{Ethyl 2-benzyl-2-[1H-indol-3- yl(phenyl)methyl]malonate (3f)}

Colorless needles: mp 135.5-136.0 ${ }^{\circ} \mathrm{C} ;{ }^{1} \mathrm{H}$ NMR (500 MHz, $\left.\mathrm{CDCl}_{3}\right) \delta 0.85(\mathrm{t}, J=7.3 \mathrm{~Hz}, 3 \mathrm{H}), 1.01(\mathrm{t}, J=7.3 \mathrm{~Hz}, 3 \mathrm{H})$, 3.43 (d, $J=14.0 \mathrm{~Hz}, 1 \mathrm{H}$ ), 3.60 (d, $J=14.0 \mathrm{~Hz}, 1 \mathrm{H}$ ), 3.844.03 (m, 4H), 5.16 (s, 1H), 6.98-7.01 (m, 3H), 7.10-7.19 (m, $7 \mathrm{H}), 7.31$ (d, $J=8.5 \mathrm{~Hz}, 1 \mathrm{H}), 7.40$ (d, $J=7.9 \mathrm{~Hz}, 3 \mathrm{H}), 7.72$ $(\mathrm{d}, J=2.4 \mathrm{~Hz}, 1 \mathrm{H}), 8.11(\mathrm{~s}, 1 \mathrm{H}) ;{ }^{13} \mathrm{C} \mathrm{NMR}(67.8 \mathrm{MHz}$, $\left.\mathrm{CDCl}_{3}\right) \delta 13.4,13.6,42.0,48.3,60.8,60.9,64.4,110.8$, 115.3, 118.8, 119.3, 121.8, 122.9, 126.5, 126.6 127.5, 127.8, 127.9, 130.2, 130.3, 135.4, 136.8, 140.4, 170.1, 171.1; IR $\left(\mathrm{CHCl}_{3}, \mathrm{~cm}^{-1}\right)$ 3480, 1717; Anal. Calcd for $\mathrm{C}_{27} \mathrm{H}_{29} \mathrm{NO}_{4}$ : C, 76.46; H, 6.42; N, 3.07. Found: C, 76.63; H, 6.51; N, 3.09.

\subsubsection{Benzyl 2-[1H-indol-3-yl(phenyl)methyl]malonate}

(3g)

White powder: mp 135.8-136.2 ${ }^{\circ} \mathrm{C} ;{ }^{1} \mathrm{H}$ NMR (500 MHz, $\left.\mathrm{CDCl}_{3}\right) \delta 4.42(\mathrm{~d}, J=12.0 \mathrm{~Hz}, 1 \mathrm{H}), 4.90-4.97(\mathrm{~m}, 4 \mathrm{H})$,
5.12 (d, $J=12.0 \mathrm{~Hz}, 1 \mathrm{H}), 6.94$ (d, $J=7.3 \mathrm{~Hz}, 2 \mathrm{H}), 7.02-$ 7.05 (m, 4H), 7.16-7.28 (m, 11H), 7.32 (d, $J=7.3 \mathrm{~Hz}, 2 \mathrm{H})$, 7.51 (d, $J=7.9 \mathrm{~Hz}, 1 \mathrm{H}), 7.87$ (s, 1H); ${ }^{13} \mathrm{C}$ NMR (125.7 $\left.\mathrm{MHz}, \mathrm{CDCl}_{3}\right) \delta 42.9,58.3,67.1,67.1,111.0,116.4,119.2$, $119.5,121.1,122.2,126.5,126.7,127.9,128.0,128.0$, $128.1,128.1,128.3,128.4,135.0,135.1,136.2,141.1$, 167.6, 167.7; IR $\left(\mathrm{CHCl}_{3}, \mathrm{~cm}^{-1}\right)$ 3478, 2386, 1755, 1732; Anal. Calcd for $\mathrm{C}_{32} \mathrm{H}_{27} \mathrm{NO}_{4}$ : C, 78.51; H, 5.56; N, 2.86 . Found: C, 78.45; H, 5.60; N, 2.92.

\subsubsection{Ethyl 2-[1H-indol-3-yl(phenyl)methyl]-3- oxobutanoate (3h) ${ }^{8 \mathrm{c}}$}

A mixture of diastereomers (83:17); white powder: mp $162-162.5{ }^{\circ} \mathrm{C}$ (from hexane/benzene $=1: 1$ ) [ref. $^{8 \mathrm{c}} 162-163$ $\left.{ }^{\circ} \mathrm{C} \quad(\mathrm{EtOH})\right] ;{ }^{1} \mathrm{H} \quad \mathrm{NMR} \quad\left(500 \mathrm{MHz}, \mathrm{CDCl}_{3}\right.$, major diastereomer) $\delta 0.97(\mathrm{t}, J=7.3 \mathrm{~Hz}, 3 \mathrm{H}), 2.04$ (s, 3H), 3.944.00 (m, 2H), 4.50 (d, $J=11.6 \mathrm{~Hz}, 1 \mathrm{H}), 5.09$ (d, $J=11.6$ Hz, 1H), 7.01-7.35 (m, 9H), 7.54 (d, $J=7.9 \mathrm{~Hz}, 1 \mathrm{H}), 8.03$ (s, $1 \mathrm{H}) ;{ }^{1} \mathrm{H}$ NMR (500 MHz, $\mathrm{CDCl}_{3}$, minor diastereomer) $\delta$ 0.99 (t, $J=7.3 \mathrm{~Hz}$, minor diastereomer $3 \mathrm{H}$ ), 2.14 (s, 3H), 3.94-4.00 (m, 2H), 4.38 (d, $J=12.3 \mathrm{~Hz}, 1 \mathrm{H}), 5.06$ (d, $J=$ 12.2 Hz, 1H), 7.01-7.35 (m, 9H), 7.54 (d, $J=7.9 \mathrm{~Hz}, 1 \mathrm{H})$, $8.06(\mathrm{~s}, 1 \mathrm{H}) ;{ }^{13} \mathrm{C} \mathrm{NMR}\left(125.7 \mathrm{MHz}, \mathrm{CDCl}_{3}\right.$, major diastereomer) $\delta 13.8,30.3,42.6,61.4,65.9,111.0,117.3$, $119.4,119.5,120.7,122.3,126.8,128.1,128.1,128.4$, 128.6, 136.2, 141.3, 168.0 .

\subsubsection{3-[1H-Indol-3-yl(phenyl)methyl]pentane-2,4-dione $(3 i)^{8 d}$}

Colorless plates: mp 153.5-154.0 ${ }^{\circ} \mathrm{C}$ (from hexane-ethyl acetate $=5: 2$ ) $\left[\right.$ lit. $^{8 \mathrm{~d}} \mathrm{mp} 150-152{ }^{\circ} \mathrm{C}$ (from EtOH) $] ;{ }^{1} \mathrm{H}$ NMR (500 MHz, $\left.\mathrm{CDCl}_{3}\right) \delta 1.93$ (s, 3H), 2.05 (s, 3H), 4.64 (d, $J=$ $12.2 \mathrm{~Hz}, 1 \mathrm{H}), 5.09$ (d, $J=12.2 \mathrm{~Hz}, 1 \mathrm{H}), 7.04(\mathrm{t}, J=7.0 \mathrm{~Hz}$, 1H), 7.11-7.16 (m, 3H), $7.23(\mathrm{t}, J=7.6 \mathrm{~Hz}, 2 \mathrm{H}), 7.30$ (t, $J$ $=7.9 \mathrm{~Hz}, 3 \mathrm{H}), 7.53(\mathrm{~d}, J=7.9 \mathrm{~Hz}, 1 \mathrm{H}), 8.09$ (brs, $1 \mathrm{H}) ;{ }^{13} \mathrm{C}$ NMR (125.7 MHz, $\left.\mathrm{CDCl}_{3}\right) \delta 27.6,31.3,43.2,75.3,111.1$, $116.6,119.0,119.8,121.2,122.6,126.3,126.8,128.1$, 128.6, 136.2, 141.3, 203.5, 204.1; IR $\left(\mathrm{CHCl}_{3}, \mathrm{~cm}^{-1}\right)$ 3478, 1721, 1696, 1356.

\subsubsection{2 -[1H-Indol-3-yl(phenyl)methyl]-1,3- diphenylpropane-1,3-dione (3j)}

White powder: mp 171-172 ${ }^{\circ} \mathrm{C} ;{ }^{1} \mathrm{H}$ NMR $(500 \mathrm{MHz}$, DMSO) $\delta 5.34(\mathrm{~d}, J=11.5 \mathrm{~Hz}, 1 \mathrm{H}), 6.89-7.02(\mathrm{~m}, 4 \mathrm{H})$, 7.07 (t, $J=7.6 \mathrm{~Hz}, 2 \mathrm{H}), 7.20$ (d, $J=7.8 \mathrm{~Hz}, 1 \mathrm{H}), 7.37-7.41$ (m, 4H), 7.50-7.57 (m, 5H), $7.61(\mathrm{~s}, 1 \mathrm{H}), 8.03$ (d, $J=7.0$ $\mathrm{Hz}, 2 \mathrm{H}), 8.08$ (d, $J=7.0 \mathrm{~Hz}, 2 \mathrm{H}), 10.8$ (brs, $1 \mathrm{H}) ;{ }^{13} \mathrm{C}$ NMR (125.7 MHz, DMSO) $\delta$ 43.7, 60.0, 111.1, 116.7, 118.3, $118.5,121.0,122.1,125.9,126.5,127.7,128.5,128.5$, 128.6, 128.6, 128.7, 133.4, 135.8, 136.3, 136.4, 142.6, 193.4, 194.4; IR $\left(\mathrm{CHCl}_{3}, \mathrm{~cm}^{-1}\right)$ 3480, 1728; HRMS (EI) Calculated for $\mathrm{C}_{30} \mathrm{H}_{23} \mathrm{O}_{2} \mathrm{~N}$ : 429.17288. Found: 429.17405 .

\subsubsection{2-Cyano-3-(1H-indol-3-yl)-3-(4-} chlorophenyl)propionic Acid Ethyl Ester (3k)

A mixture of diastereomers (50:50); colorless oil: ${ }^{1} \mathrm{H}$ NMR $\left(500 \mathrm{MHz}, \mathrm{CDCl}_{3}\right) \delta 1.09(\mathrm{t}, J=7.3 \mathrm{~Hz}, 3 \mathrm{H}), 1.13(\mathrm{t}, J=$ $7.3 \mathrm{~Hz}, 3 \mathrm{H}), 4.07-4.16(\mathrm{~m}, 5 \mathrm{H}), 4.31$ (d, $J=6.1 \mathrm{~Hz}, 1 \mathrm{H})$, 5.02 (d, $J=6.7 \mathrm{~Hz}, 1 \mathrm{H}), 5.08$ (d, $J=6.1 \mathrm{~Hz}, 1 \mathrm{H}), 7.01-7.10$ (m, 2H), 7.17-7.38 (m, 15H), 7.46 (d, $J=2.4 \mathrm{~Hz}, 1 \mathrm{H}), 8.23$ (s, $1 \mathrm{H}), 8.28(\mathrm{~s}, 1 \mathrm{H}) ;{ }^{13} \mathrm{C}$ NMR $\left(125.7 \mathrm{MHz}, \mathrm{CDCl}_{3}\right) \delta 13.7$, 
13.8, 42.4, 42.5, 44.0, 44.8, 63.0, 111.3, 111.4, 112.8, 114.3, $115.9,116.0,118.6,118.8,119.9,120.0,122.0,122.1$, $122.7,122.8,125.9,126.4,128.9,129.0,129.4,129.8$, 133.6, 133.8, 136.1, 136.2, 137.1, 138.2, 165.0, 165.0; IR $\left(\mathrm{CHCl}_{3}, \mathrm{~cm}^{-1}\right)$ 3476, 1745, 1223; HRMS (EI) Calculated for $\mathrm{C}_{20} \mathrm{H}_{17} \mathrm{~N}_{2} \mathrm{O}_{2} \mathrm{Cl}$ : 352.09786. Found 352.09807.

\subsubsection{2-Cyano-3-(1H-indol-3-yl)-3-(4- methoxyphenyl)propionic Acid Ethyl Ester (3l)}

A mixture of diastereomers (50:50); white powder: mp 128-128.5 ${ }^{\circ} \mathrm{C}$; ${ }^{1} \mathrm{H}$ NMR (500 MHz, $\left.\mathrm{CDCl}_{3}\right) \delta 1.08(\mathrm{t}, J=$ $7.0 \mathrm{~Hz}, 3 \mathrm{H}), 1.12$ (t, $J=7.0 \mathrm{~Hz}, 3 \mathrm{H}), 3.74(\mathrm{~s}, 3 \mathrm{H}), 3.75$ (s, 3H), 4.07-4.16 (m, 5H), 4.30 (d, $J=6.1 \mathrm{~Hz}, 1 \mathrm{H}), 4.99$ (d, $J$ $=6.1 \mathrm{~Hz}, 1 \mathrm{H}), 5.05(\mathrm{~d}, J=6.7 \mathrm{~Hz}, 1 \mathrm{H}), 6.80-6.84(\mathrm{~m}, 4 \mathrm{H})$, 7.00 (t, $J=7.3 \mathrm{~Hz}, 1 \mathrm{H}), 7.04$ (t, $J=7.3 \mathrm{~Hz}, 1 \mathrm{H}), 7.15-7.36$ (m, 11H), 7.45 (d, $J=2.4 \mathrm{~Hz}, 1 \mathrm{H}), 8.21$ (brs, $1 \mathrm{H}$ ), 8.25 (brs, $1 \mathrm{H}) ;{ }^{13} \mathrm{C}$ NMR (125.7 MHz, $\left.\mathrm{CDCl}_{3}\right) \delta$ 13.7, 13.8, 42.4, 42.5, 44.5, 45.2, 55.2, 55.2, 62.8, 62.8, 111.2, 111.3, 113.6, $114.0,114.1,115.1,116.2,116.4,118.8,119.0,119.8$, $121.9,122.0,122.4,122.5,126.1,126.6,128.5,128.6$, $128.9,129.0,129.1,129.5,130.7,131.8,136.1,136.3$, 159.0, 159.1, 165.3, 165.3; IR $\left(\mathrm{CHCl}_{3}, \mathrm{~cm}^{-1}\right)$ 3478, 2253, 1744, 1512, 1252; HRMS (EI) Calculated for $\mathrm{C}_{21} \mathrm{H}_{20} \mathrm{O}_{3} \mathrm{~N}_{2}$ : 348.14740. Found: 348.14812.

\subsubsection{2-Cyano-3-(1H-indol-3-yl)-5-phenylpenta-4-enoic Acid Ethyl Ester (3m)}

A mixture of diastereomers (60:40); colorless oil: ${ }^{1} \mathrm{H}$ NMR (500 MHz, $\mathrm{CDCl}_{3}$, major diastereomer) $\delta 1.20$ (t, $J=7.0$ $\mathrm{Hz}, 3 \mathrm{H}), 4.05-4.08$ (m, 1H), 4.21 (q, $J=7.0 \mathrm{~Hz}, 2 \mathrm{H}), 4.60-$ $4.63(\mathrm{~m}, 1 \mathrm{H}), 6.51-6.70(\mathrm{~m}, 2 \mathrm{H}), 7.11-7.39$ (m, 9H), 7.64 (d, $J=7.9 \mathrm{~Hz}, 1 \mathrm{H}), 8.23$ (brs, $1 \mathrm{H})$; ${ }^{1} \mathrm{H}$ NMR $\left(500 \mathrm{MHz}, \mathrm{CDCl}_{3}\right.$, minor diastereomer) $\delta 1.06(\mathrm{t}, J=7.0 \mathrm{~Hz}, 3 \mathrm{H}), 4.05-4.08$ (m, 3H), 4.60-4.63 (m, 1H), 6.51-6.70 (m, 2H), 7.11-7.39 $(\mathrm{m}, 9 \mathrm{H}), 7.64$ (d, $J=7.9 \mathrm{~Hz}, 1 \mathrm{H}), 8.26(\mathrm{~s}, 1 \mathrm{H}) ;{ }^{13} \mathrm{C} \mathrm{NMR}$ (125.7 $\mathrm{MHz}, \mathrm{CDCl}_{3}$, mixture of diastereomers) $\delta 13.7,14.0$, 41.2, 41.3, 44.6, 44.7, 62.8, 62.8, 111.4, 111.6, 112.6, 113.7, $115.8,116.1,118.3,118.8,119.9,120.0,122.2,122.4$, $122.5,122.7,125.5,125.6,126.2,126.5,126.6,127.5$, $127.9,127.9,128.6,132.6,133.8,136.1,136.2,136.3$, 136.4, 165.2; IR $\left(\mathrm{CHCl}_{3}, \mathrm{~cm}^{-1}\right) 3478,2359,1745$; HRMS (EI) Calculated for $\mathrm{C}_{22} \mathrm{H}_{20} \mathrm{~N}_{2} \mathrm{O}_{2}$ : 344.15248. Found 344.15233.

\subsubsection{2-Cyano-3-(1H-indol-3-yl) penta-4-enoic Acid Ethyl Ester (3n)}

A mixture of diastereomers (60:40); colorless oil: ${ }^{1} \mathrm{H}$ NMR (500 MHz, $\mathrm{CDCl}_{3}$, major diastereomer) $\delta 1.24$ (t, $J=7.0$ $\mathrm{Hz}, 3 \mathrm{H}), 3.99$ (d, $J=6.1 \mathrm{~Hz}, 1 \mathrm{H}), 4.22$ (q, $J=7.0 \mathrm{~Hz}, 2 \mathrm{H})$, 4.44-4.47 (m, 1H), 5.25-5.37 (m, 2H), 6.13-6.28 (m, 1H), 7.11-7.24 (m, 3H), 7.38 (d, $J=7.9 \mathrm{~Hz}, 1 \mathrm{H}), 7.59$ (t, $J=8.2$ $\mathrm{Hz}, 1 \mathrm{H}), 8.20$ (brs, $1 \mathrm{H})$; ${ }^{1} \mathrm{H}$ NMR (500 MHz, $\mathrm{CDCl}_{3}$, minor diastereomer) $\delta 1.08(\mathrm{t}, J=7.0 \mathrm{~Hz}, 3 \mathrm{H}), 3.99(\mathrm{~d}, J=6.1 \mathrm{~Hz}$, $1 \mathrm{H}), 4.07$ (q, $J=7.0 \mathrm{~Hz}, 2 \mathrm{H}), 4.44-4.47$ (m, $1 \mathrm{H}), 5.25-5.37$ (m, 2H), 6.13-6.28 (m, 1H), 7.11-7.24 (m, 2H), 7.31 (d, $J=$ $2.4 \mathrm{~Hz}, 1 \mathrm{H}), 7.36$ (d, $J=7.9 \mathrm{~Hz}, 1 \mathrm{H}), 7.59$ (t, $J=8.2 \mathrm{~Hz}$, 1H), 8.23 (brs, $1 \mathrm{H}) ;{ }^{13} \mathrm{C}$ NMR $\left(125.7 \mathrm{MHz}, \mathrm{CDCl}_{3}\right.$, mixture of diastereomers) $\delta 13.7,13.9,41.6,44.2,44.3,111.4$, $111.5,112.2,113.4,115.6,116.0,117.5,118.2,118.7$, $118.8,119.7,119.9,122.2,122.4,122.5,122.6,125.6$, 126.2, 134.3, 136.0, 136.2, 136.3, 165.2, 165.3; IR $\left(\mathrm{CHCl}_{3}\right.$, $\mathrm{cm}^{-1}$ ) 3478, 2361, 1743; HRMS (EI) Calculated for $\mathrm{C}_{16} \mathrm{H}_{16} \mathrm{~N}_{2} \mathrm{O}_{2}$ : 268.12118. Found 268.12117.

\subsection{Typical experimental procedure for oxidative C-C bond formation of 3-benzylindole 5 a with higher-order cyanocuprates (Table 3 , entry 4 )}

A solution of $\mathrm{Ph}_{2} \mathrm{Cu}(\mathrm{CN}) \mathrm{Li}_{2}$ was prepared as follows; to a stirred solution of copper(I) cyanide (61.2 mg, $0.68 \mathrm{mmol}$ ) in $\mathrm{Et}_{2} \mathrm{O}(4 \mathrm{~mL})$ was added dropwise a solution of phenyllithium (1.14 $\mathrm{N}$ in cyclohexane-ether, $1.06 \mathrm{ml}, 1.21$ mmol) at $-20{ }^{\circ} \mathrm{C}$ for $1 \mathrm{~h}$.

To a stirred solution of 5 a (50 mg, $0.24 \mathrm{mmol}$ ) in THF (3 mL) was added dropwise a solution of $n$-BuLi (1.61 $N$ in hexane, $0.20 \mathrm{~mL}, 0.32 \mathrm{mmol}$ ) at $-78{ }^{\circ} \mathrm{C}$. After the mixure was stirred at the same temperature for $15 \mathrm{~min}$, a solution of 4 (83 $\mathrm{mg}, 0.38 \mathrm{mmol})$ in THF (1 mL) was added at -78 ${ }^{\circ} \mathrm{C}$. After the mixture was stirred at the same temperature for $30 \mathrm{~min}$, a solution of $\mathrm{Ph}_{2} \mathrm{Cu}(\mathrm{CN}) \mathrm{Li}_{2}$ was added at -78 ${ }^{\circ} \mathrm{C}$ with a cannula, and the reaction mixture was stirred at the same temperature for $30 \mathrm{~min}$. the product 9d was obtained as usual manner.

\subsubsection{3-(1-Phenylethyl)indole (9a) ${ }^{5 a}$}

White solid; ${ }^{1} \mathrm{H}$ NMR (500 MHz, $\left.\mathrm{CDCl}_{3}\right) \delta 1.70(\mathrm{t}, J=7.3$ $\mathrm{Hz}, 3 \mathrm{H}), 4.37$ (q, $J=7.3 \mathrm{~Hz}, 1 \mathrm{H}), 6.98-7.37$ (m, 10H), 7.96 (s, $1 \mathrm{H}) ;{ }^{13} \mathrm{C}$ NMR $\left(125.7 \mathrm{MHz}, \mathrm{CDCl}_{3}\right) \delta 22.4,36.9,111.0$, 119.2 , 119.7, 121.0, 121.9, 122.3, 125.9, 127.4, 128.3, 128.7, 136.6, 146.8 .

\subsubsection{3-(1-Phenylpentyl)indole (9b)}

Colorless plates: mp 74.5-75.5 ${ }^{\circ} \mathrm{C}$; ${ }^{1} \mathrm{H}$ NMR (270 MHz, $\left.\mathrm{CDCl}_{3}\right) \delta 0.86(\mathrm{t}, J=7.0 \mathrm{~Hz}, 3 \mathrm{H}), 1.27-1.35(\mathrm{~m}, 4 \mathrm{H}), 1.96-$ $2.03(\mathrm{~m}, 1 \mathrm{H}), 2.14-2.21(\mathrm{~m}, 1 \mathrm{H}), 4.14$ (t, $J=7.6 \mathrm{~Hz}, 1 \mathrm{H})$, 6.97-7.02 (m, 2H), 7.11 (d, $J=8.1 \mathrm{~Hz}, 1 \mathrm{H}), 7.15$ (d, $J=7.8$ Hz, 1H), 7.23-7.30 (m, 5H), 7.44 (d, $J=8.1 \mathrm{~Hz}, 1 \mathrm{H}), 7.83$ (s, $1 \mathrm{H}) ;{ }^{13} \mathrm{C}$ NMR $\left(125.7 \mathrm{MHz}, \mathrm{CDCl}_{3}\right) \delta 14.0,22.8,30.3$, 35.9, 42.9, 111.0, 119.2, 119.5, 120.7, 120.9, 121.9, 125.8, 127.1, 127.9, 128.2, 136.5, 145.6; IR $\left(\mathrm{CHCl}_{3}, \mathrm{~cm}^{-1}\right) 3481$, 1456; HRMS (EI) Calculated for $\mathrm{C}_{21} \mathrm{H}_{19} \mathrm{~N}$ : 263.16740 . Found 263.16743.

\subsubsection{3-(2,2-Dimethyl-1-phenylpropyl)indole (9c)}

White powder: mp 87.5-88.5 ${ }^{\circ} \mathrm{C}$; ${ }^{1} \mathrm{H}$ NMR (270 MHz, $\left.\mathrm{CDCl}_{3}\right) \delta 1.04(\mathrm{~s}, 9 \mathrm{H}), 4.15(\mathrm{~s}, 1 \mathrm{H}), 7.02-7.40(\mathrm{~m}, 9 \mathrm{H})$, 7.58 (d, $J=7.7 \mathrm{~Hz}, 1 \mathrm{H}), 7.98$ (s, $1 \mathrm{H}) ;{ }^{13} \mathrm{C}$ NMR $(67.8 \mathrm{MHz}$, $\left.\mathrm{CDCl}_{3}\right) \delta$ 29.0, 35.2, 53.6, 110.7, 117.8, 119.0 119.1, 121.0, 121.8, 125.7, 127.5, 128.8, 130.0, 135.1, 143.3; IR $\left(\mathrm{CHCl}_{3}\right.$, $\mathrm{cm}^{-1}$ ) 3481, 1456; HRMS (EI) Calculated for $\mathrm{C}_{21} \mathrm{H}_{19} \mathrm{~N}$ : 263.16740. Found 263.16682.

\subsubsection{3-Diphenylmethylindole (9d) ${ }^{21}$}

White needles: mp $124.5-124.8{ }^{\circ} \mathrm{C}$ (lit. ${ }^{21} 121{ }^{\circ} \mathrm{C}$ ); ${ }^{1} \mathrm{H}$ NMR $\left(500 \mathrm{MHz}, \mathrm{CDCl}_{3}\right) \delta 5.66(\mathrm{~s}, 1 \mathrm{H}), 6.54(\mathrm{~d}, J=2.2 \mathrm{~Hz}, 1 \mathrm{H})$, 6.96-6.99 (m, 1H), 7.14-7.28 (m, 12H), 7.33 (d, $J=8.1 \mathrm{~Hz}$, 1H), $7.88(\mathrm{~s}, 1 \mathrm{H}) ;{ }^{13} \mathrm{C}$ NMR (125.7 MHz, $\left.\mathrm{CDCl}_{3}\right) \delta 48.8$, $111.0,119.4,119.9,122.1,124.0,126.2,127.0,128.3$, $129.0,136.7,143.9$. 


\section{Acknowledgments}

The authors are grateful for the financial support from Takeda Science Foundation, the Research Foundation for Pharmaceutical Sciences and the Sasakawa Scientific Research Grant from The Japan Science Society, and a Grant-in-Aid for Scientific Research from the Ministry of Education, Culture, Sports, Science, and Technology of Japan.

\section{References}

1. (a) Sundberg, R. Indoles, Academic Press: San Diego, 1996; (b) Robinson, B. The Fischer Indole Synthesis, WileyInterscience: New York, 1982; (c) Sundberg, R. The Chemistry of Indoles, Academic Press: New York, 1970.

2. For reviews on the synthesis of indoles: (a) Humphrey, R. R.; Kuethe, J. T. Chem. Rev. 2006, 106, 2875-2911; (b) Gilchrist, T. L. J. Chem. Soc., Perkin Trans. 1 2001, 2491-2515; (c) Gribble, G. W. J. Chem. Soc., Perkin Trans. 1 2000, 10451075; (d) Pindur, U.; Adam, R. J. Heterocyclic Chem. 1988, 25, $1-8$.

3. (a) Snyder, H.; Smith, C.; Stewart, J. J. Am. Chem. Soc. 1944, 66, 200-204; (b) Albright, J. D.; Snyder, H. R. J. Am. Chem. Soc. 1959, 81, 2239-2245; (c) Howe, E. E.; Zambito, A. J.; Snyder, H. R.; Tishler, M. J. Am. Chem. Soc. 1945, 67, 38-39; (d) Albertson, N. F.; Archer, S.; Suter, C. M. J. Am. Chem. Soc. 1945, 67, 36-37; (e) Somei, M.; Karasawa, Y.; Kaneko, C. Heterocycles 1981, 16, 941-949.

4. Review: Semenov, B. B.; Granik, V. G. Pharm. Chem. J., 2004, 38, 287-310.

5. (a) Ballini, R.; Palmieri, A.; Petrini, M.; Torregiani, E. Org. Lett. 2006, 8, 4093-4096; (b) Palmieri, A.; Petrini, M. J. Org. Chem. 2007, 72, 1863-1866.

6. (a) Runti, C. Gazz. Chim. Ital. 1951, 81, 613-620; (b) Runti, C.; Orlando, G. Ann. Chim. (Rome) 1953, 43, 308-314.

7. Poppelsdorf, F.; Holt, S. J. J. Chem. Soc. 1954, 4094-4101.

8. (a) Semenov, B. B.; Novikov, K. A.; Lysenko, K. A.; Kachala, V. V. Tetrahedron Lett. 2006, 47, 3479-3483; (b) Semenov, B. B.; Smushkevich, V. V. Y. I.; Levina, I. I.; Kurkovskaya, L. N.; Lysenko, K. A.; Kachala, V. V. Chem. Heterocycl. Compounds 2005, 41, 730-738; (c) Semenov, B. B.; Smushkevich, Y. I.; Grintselev-Knyazev, G. V.; Antipin, M. Y. Russ. Chem. Bull. Int. Ed. 2001, 50, 570-571; (d) Semenov, B. B.; Smushkevich, Y. I. Russ. Chem. Bull. Int. Ed. 2001, 50, 543-545.
9. Recent examples: (a) Gibbs, T. J. K.; Tomkinson, N. C. O. Org. Biomol. Chem. 2005, 3, 4043-4045; (b) Shirakawa, S.; Kobayashi, S. Org. Lett. 2006, 8, 4939-4942; (c) Ke, B.; Qin, Y.; He, Q.; Huang, Z.; Wang, F. Tetrahedron Lett. 2005, 46, 1751-1753.

10. (a) Schellenberg, K. A. J. Biol. Chem. 1965, 240, 1165-1169;

(b) Schellenberg, K. A. J. Biol. Chem. 1967, 242, 1815-1820;

(c) Schellenberg, K. A.; McLean, G. W. J. Am. Chem. Soc. 1966, 88, 1077-1079; (d) Schellenberg, K. A.; McLean, G. W.; Lipton, H. L.; Lietman, P. S. J. Am. Chem. Soc. 1967, 89, 1948-1950; (e) Huffman, R. W.; Bruice, T. C. J. Am. Chem. Soc. 1967, 89, 6243-6251.

11. Burr, G. O.; Gortner, R. A. J. Am. Chem. Soc. 1924, 46, 1224-1246.

12. Recent reports: (a) Catino, A. J.; Nicholas, J. M.; Nettles, B. J.; Doyle, M. P. J. Am. Chem. Soc. 2006, 128, 5648-5649; (b) Murahashi, S.-I.; Komiya, N.; Terai, H. Angew. Chem., Int. Ed. 2005, 44, 6931-6933; (c) Li, Z.; Li, C.-J. J. Am. Chem. Soc. 2005, 127, 3672-3673 and references are cited therein.

13. (a) Matsuo, J. J. Synth. Org. Chem. Jpn. 2004, 62, 574-583; (b) Mukaiyama, T. Angew. Chem., Int. Ed. 2004, 43, 55905614.

14. (a) Matsuo, J.; Tanaki, Y.; Ishibashi, H. Org. Lett. 2006, 8, 4371-4374; (b) Matsuo, J.; Tanaki, Y.; Ishibashi, H. Tetrahedron Lett. 2007, 48, 3233-3236; (c) Matsuo, J.; Kawai, H.; Ishibashi, H. Tetrahedron Lett. 2007, 48, 3155-3158.

15. For application to other one-pot C-C bond formations, see: (a) Matsuo, J.; Aizawa, Y. Chem. Commun. 2005, 2399-2401; (b) Matsuo, J.; Shibata, T.; Kitagawa, H.; Mukaiyama, T. Arkivoc 2001, 58-65; (c) Kerr, W. J.; Pearson, C. M.; Thurston, G. J. Org. Biomol. Chem. 2006, 4, 47-50; (d) Crawford, J. J.; Henderson, K. W.; Kerr, W. J. Org. Lett. 2006, 8, 5073-5076.

16. Whitney, S.; Grigg, R.; Derrick, A.; Keep, A. Org. Lett. 2007, 9, 3299-3302.

17. Kimura, M.; Futamata, M.; Mukai, R.; Tamaru, Y. J. Am. Chem. Soc. 2005, 127, 4592-4593.

18. (a) Nickel-catalyzed reaction: Pratt, E. T.; Botimer, L. W. J. Am. Chem. Soc. 1957, 79, 5248-5250; (b) Wenkert, E.; Angell, E. C.; Ferreira, V. F.; Michelotti, E. L.; Piettre, S. R.; Sheu, J.-H.; Swindell, C. S. J. Org. Chem. 1986, 51, 23432351.

19. Matsuo, J.; Iida, D.; Tatani, K.; Mukaiyama, T. Bull. Chem. Soc. Jpn. 2002, 75, 223-234.

20. Ernest, F. P.; Laurence, W. B. J. Am. Chem. Soc. 1957, 79, 5248-5250.

21. Yasuda, M.; Somyo, T.; Baba, A. Angew. Chem., Ind. Ed. 2006, 45, 793-796. 